\title{
Creating Single Boundary between Two CdTe (111) Wafers with Controlled Orientation by Wafer Bonding
}

\author{
Ce Sun ${ }^{1}$, Ning Lu ${ }^{1}$, Guoda Lian ${ }^{1}$, Jinguo Wang ${ }^{1}$, Xin Peng ${ }^{1}$, Robert F. Klie ${ }^{2}$, and Moon J. Kim ${ }^{1}$ \\ ${ }^{1 .}$ Department of Materials Science and Engineering, The University of Texas at Dallas, \\ Richardson, Texas 75080, USA \\ ${ }^{2}$ Department of Physics, University of Illinois at Chicago, Chicago, Illinois 60607, USA
}

Zinc-blende (ZB) CdTe has drawn great attention as optoelectronic and solar energy conversion materials since it has a near optimum band gap of $1.6 \mathrm{eV}$ and a absorption coefficient greater than $5 \times 10^{5} / \mathrm{cm}$. CdTe can be either ZB or wurtzite (WZ) structures, resulting in different electronic properties. It has been reported that the twin superlattice with numerous twin boundaries in III-V and II-VI semiconductor nanowires along $<111>$ growth orientation of ZB structures considerably enhance band gap engineering and mechanical behavior in quasi-one-dimensional materials [1]. This opens new possibilities for properties and functionalities at the atomic and quantum scales by controlling the twin boundary and modulating twin densities. Therefore, it is an in-demand and challenging feat to "create" a single twin boundary in a nanowire or bulk material in order to understand the electronic and mechanical characteristics of the III-V and II-VI quantum well or barrier. Wafer bonding, which enables the direct integration of two or more single crystal wafers with controlled surfaces and orientation, is a key technique in creating a single boundary [2]. In this study, we show the creation of a single boundary between two identical CdTe single crystals.

Single-side polished $5 \times 5 \mathrm{~mm}^{2}$ p-type CdTe (111) wafers with a thickness of $500 \mu \mathrm{m}$ were used for the bonding experiments. Out of plane $(2 \theta / \omega$ scan) and in-plane ( $\varphi$ scan) XRD were used to determine the crystal orientations of the planes and flats. Surface roughness (RMS) was measured by AFM in tapping mode. For the cleaning process, samples were rinsed in de-ionized water for $5 \mathrm{~s}$, dipped in $\mathrm{HCl}(15 \%)$ for $30 \mathrm{~s}$, and then rinsed in de-ionized water for $5 \mathrm{~s}$. The wafer pair was loaded into a vacuum bonder immediately after drying under a flow of nitrogen gas to prevent oxidation of the CdTe surface. Bonding was performed at $400{ }^{\circ} \mathrm{C}$ for $20 \mathrm{hrs}$ under a pressure of $1 \mathrm{MPa}$. Interface morphology and atomic arrangement were analyzed using HRTEM and HAADF-STEM. Electrical characteristics across the twin boundary were compared against those of single crystal CdTe.

Three types of boundaries were created without an amorphous layer at the interface by controlling the relative rotation angle of the two bonded parts. Figure 1(a) shows the HRTEM of the bonded pair with a relative rotation angle of $180^{\circ}$. The bonded interface is a twin boundary along the $<0 \overline{1} 1>$ zone axis. The diffraction pattern from the interface, as shown in Figure 1(b), has confirmed that the bonded boundary is a (111) [ $\left.\begin{array}{lll}0 & \overline{1} & 1\end{array}\right]$ twin boundary [3]. A small misorientation angle from the perfect $180^{\circ}$ rotation angle may exist due to a slight misalignment of the bonded pair; the measured rotation angle was $177.6^{\circ}$. Figure 2 presents the HAADF-STEM images of the two bonded portions, and shows that the twin boundary was one unit layer of WZ structure between ZB matrices. The Cd-Te dumbbell structure was observed along $<011>$, which could be used to identify atom species at the termination of the atomic planes. The intensity profiles from HAADF images demonstrated that the arrangement of Cd-Te dumbbell were $\mathrm{Cd}$ - and Te- terminated on the left and right portions, respectively, as shown in Figure 2(c) and (d). Our preliminary I-V measurement shows a potential barrier at the bonded interface, indicating that the one unit layer of WZ structure between ZB matrices may act as a quantum barrier [3]. 
Two other types of bonded boundaries were created with a designed relative rotation angle of $90^{\circ}$ and $0^{\circ}$, as shown in Figures 3 and 4, respectively. The crystallographic orientations of the two bonded pairs

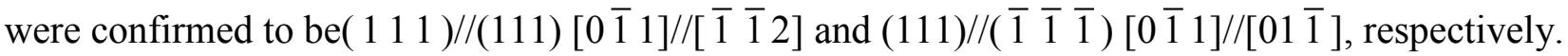

\section{References:}

[1] EL Wood, F Sansoz, Nanoscale 4 (2012) p. 5268.

[2] M Alexw, U Gosele in "Wafer Bonding" (Springer, 2004).

[3] C Sun et al, Appl Phys Lett 103 (2013) p. 252104.

[4] This work was supported by Leading Foreign Research Institute Recruitment Program through NRF funded by MSIP, Korea (2013K1A4A3055679) and the DOE BRIDGE (DE-EE0005956).

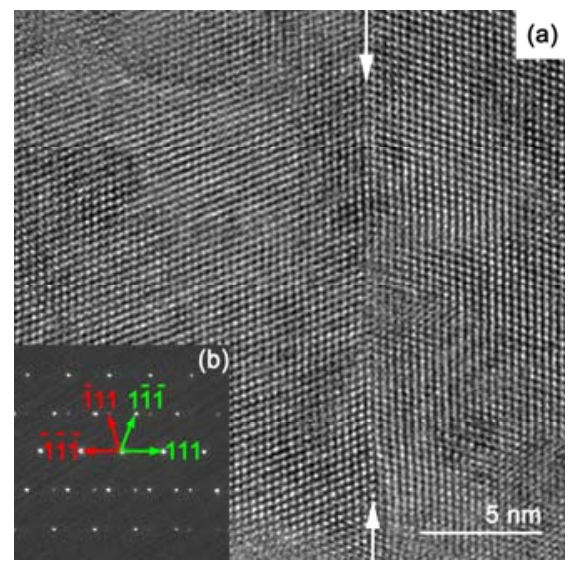

Figure 1. TEM image (a) of the bonded pair shows the twin boundary with a coherent twin interface. The interface is indicated by the two white arrows. Diffraction patterns for the interface (b) of the bonded pair indicated that the crystallographic orientation relationship of the bonded pair was $(111) / /(111)$ and $\left[\begin{array}{lll}0 & \overline{1} & 1\end{array}\right]$ $/ /[01 \overline{1}]$.

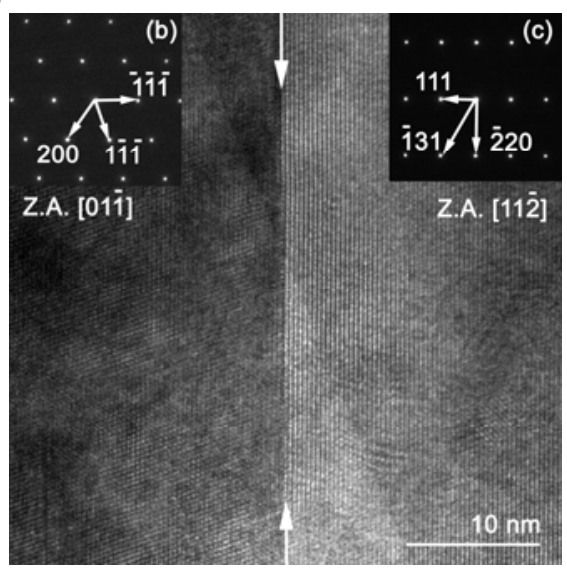

Figure 3. TEM image (a) and diffraction patterns for the left (b), and right (c) portions of the bonded pair with a relative rotation angle of $90^{\circ}$.

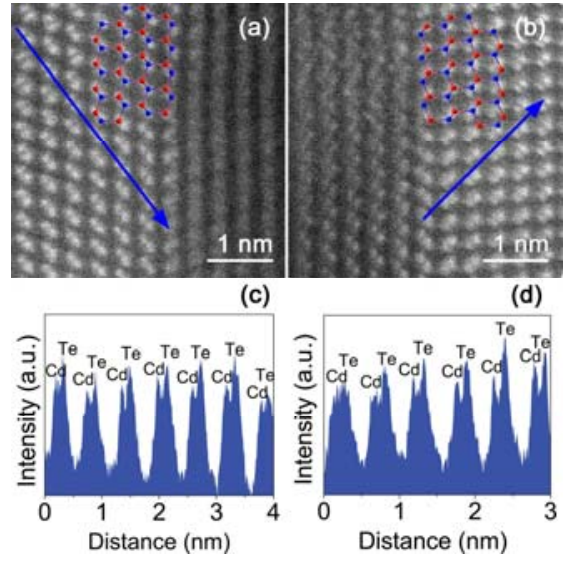

Figure 2. The $<011>$ cross-sectional HAADF-STEM images, (a) and (b) reveals the twin boundary at the bonded interface. HAADF intensity profiles (c) and (d) along the left and right parts reveal the atomic arrangement of the bonded interface. Blue dot depicts Cd. Red dot depicts Te.

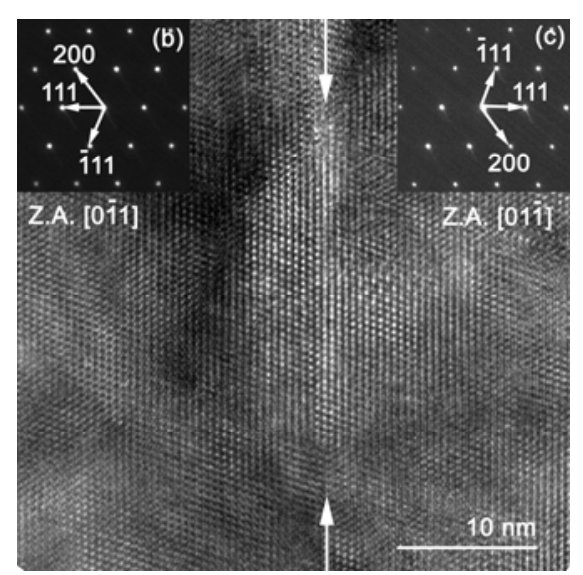

Figure 4. TEM image (a) and diffraction patterns for the left (b), right (c) portions of the bonded pair with a relative rotation angle of $0^{\circ}$. 